\title{
Finite Difference Solution to a Nonlinear Time-Fractional Logistic Model
}

\author{
Yuanyuan Yang ${ }^{1}$, Gongsheng $\mathrm{Li}^{1}$ \\ ${ }^{1}$ School of Mathematics and Statistics, Shandong University of Technology, Zibo, China \\ Correspondence: Gongsheng Li, School of Mathematics and Statistics, Shandong University of Technology, Zibo, China
}

Received: January 27, 2021 Accepted: March 16, 2021 Online Published: March 21, 2021

doi:10.5539/jmr.v13n2p60 URL: https://doi.org/10.5539/jmr.v13n2p60

\begin{abstract}
We set forth a time-fractional logistic model and give an implicit finite difference scheme for solving of the model. The $L^{2}$ stability and convergence of the scheme are proved with the aids of discrete Gronwall inequality, and numerical examples are presented to support the theoretical analysis.
\end{abstract}

Keywords: fractional logistic equation, nonlinear term, finite difference scheme, stability and convergence

\section{Introduction}

Logistic equation with space distribution is an important mathematical model in describing space population ecology with carrying capacity, which is given by Brauer \& Castillo-Chavez (2012)

$$
u_{t}=\nabla \cdot(D \nabla u)+r u(1-u / K)
$$

where $u=u(x, t)$ denotes the population density at $t>0$ and $x \in \Omega, \Omega$ is a bounded domain with smooth boundary, and $D$ is the diffusion coefficient(tensor), $r>0$ is the intrinsic growth rate, and $K>0$ is the carrying capacity of the population. Eq.(1.1) is also called Fisher equation in the research field of nonlinear partial differential equations.

There are a lot of researches on nonlinear reaction-diffusion systems like Eq.(1.1), and most of the studies focus on dynamics analysis from the viewpoints of population ecology and epidemiology, see Bai \& Wang (2005), Balanov et al. (2008), Chakraborty et al. (2007), Jin \& Zhao (2008), Korobenko \& Braverman (2009), Wang et al. (2018), Zhao (2003) for instance. On the aspect of numerical methods for nonlinear partial differential equations, based on the classical work by Xu (1994, 1996), a two-grids finite difference algorithm for nonlinear parabolic-type equations was set forth by Dawson et al. (1998), and an optimal error estimate was obtained which gave ideas and approaches for solving nonlinear fractional diffusion equation. We refer to the monograph by Sun (2018) and references therein for difference methods of solving nonlinear evolution equations.

The fractional differential equations and their applications have attracted much attentions in science and engineering during the last decades. We refer to El-Nabulsi (2011, 2019, 2020), Lima et al. (2007), Magin (2006), Moshrefi-Torbati \& Hammond (1998), Singla \& Gupta (2016) for the applications of fractional calculus in quantum mechanics, molecular physics, fluid and microfilaments, bioengineering, signal processing and system control, etc.. Especially for solute transport behaviors with memory in heterogeneous porous media with low-permeability, the fractional diffusion equations could be more suitable than those of classical diffusion equations, see Chen et al. (2010), Kelly \& Meeschaert (2019), Schumer \& Benson (2003), Zhang et al. (2009), Zhou et al. (2019) for instance. Under the constraints of available resources in a living environment, if considering the influence of historical factors on the diffusion and growth of a population, we can get a time-fractional diffusion equation by replacing the integer-order derivative of $u_{t}$ in Eq.(1.1) with a time fractional derivative, named the fractional logistic model, which is given by

$$
R \partial_{t}^{\alpha} u=\nabla \cdot(D \nabla u)+r u(1-u / K),
$$

where $R>0$ denotes the time-scale factor, and $\partial_{t}^{\alpha} u$ denotes the Caputo's fractional derivative of $u$ on $t>0$ with the order of $\alpha \in(0,1)$ (Podlubny, 1999), and the others denote the same meanings as in Eq.(1.1).

In contrast to the classical diffusion equations characterized by the exponential decay in time and Gaussian profile in space, it reveals that fractional diffusion equations possess properties of slow decay in time and long-tailed profile in space. Thus the fractional diffusion equation Eq.(1.2) could be more suitable than the classical logistic models in modeling anomalous diffusion and sub-growth of a population with carrying capacity. Nevertheless, it is very difficult to study well-posedness 
of nonlinear fractional diffusion equations like (1.2). In the case of the diffusion coefficient and the initial distribution are both constants, the unique existence of weak solution to the time-fractional diffusion equation with a nonlinear term is proved by Luchko et al. (2013), and most of the researches on nonlinear fractional differential equations are concerned with numerical methods, see Liu et al. (2015, 2016, 2018), Li \& Rui (2017) for instance. Recently, a two-grids algorithm based on finite difference method was utilized to solve a 2D nonlinear time fractional mobile-immobile solute transport model, and the $L^{2}$ stability and convergence were proved (Qiu et al., 2020), and a multi-grids algorithm was set forth for solving nonlinear fractional diffusion equations in $1 \mathrm{D}$ and $2 \mathrm{D}$ cases, and the unique solvability, stability and convergence of the numerical schemes were proved by the optimal error estimates (Maurya et al., 2021). For the detailed numerical methods of solving fractional differential equations, see the monograph (Sun \& Gao, 2020) and the references therein.

This paper is to deal with the time-fractional logistic diffusion model (1.2) in 1D case with a general initial distribution from numerics. The motivation is to get an effective method for solving of the nonlinear time-fractional logistic model which can give basis for its application in ecology and biology. We are to set forth an implicit finite difference scheme for solving of the model, and prove its stability and convergence in $L^{2}$ norm, and present numerical examples to support the theoretical analysis. As compared with the known results, the fractional model is concrete due to the special nonlinear term, and the convergent rate of the difference scheme can reach to the order of $h^{2+\alpha}$ in the case of $\tau=h^{2}$, where $\tau$ is the time step and $h$ is the space step, and $\alpha \in(0,1)$ is the order of the time fractional derivative. The rest of the paper is arranged as follows.

In section 2, an implicit finite difference scheme for the nonlinear fractional logistic problem is set forth, and in section 3 the $L^{2}$ stability and convergence are proved with the aids of discrete Gronwall inequality. Numerical examples are presented in section 4 and concluding remarks are given in section 5 .

\section{The Finite Difference Scheme}

\subsection{The Forward Problem}

Let $L>0, T>0$, and denote $\Omega=(0, L), \Omega_{T}=\Omega \times(0, T)$. Consider the 1D time-fractional logistic equation with constant diffusion coefficient:

$$
\partial_{t}^{\alpha} u=D u_{x x}+r u(1-u / K)+\gamma(x, t),(x, t) \in \Omega_{T},
$$

where $\gamma(x, t)$ is the force term. Given the initial distribution

$$
u(x, 0)=u_{0}(x), 0 \leq x \leq L,
$$

and the homogeneous Neumann boundary condition

$$
\left.\frac{\partial u}{\partial x}\right|_{x=0}=\left.\frac{\partial u}{\partial x}\right|_{x=L}=0,0 \leq t \leq T,
$$

where $u_{0}(x)$ is assumed to be a smooth function taking nonnegative values in $\Omega$.

The forward problem is composed by Eq.(2.1) with the initial boundary conditions (2.2)-(2.3). This paper is devoted to the finite difference solution of the forward problem. In the follows for convenience of writing, we denote the nonlinear source term by

$$
f(u):=r u(1-u / K) .
$$

By the real-life meanings of logistic models, the solution $u$ is nonnegative and bounded, i.e., there is $0 \leq u(x, t)<K$ for $(x, t) \in \Omega_{T}$, where $K>0$ is the carrying capacity. Obviously there exists constant $C>0$ such that the nonlinear function $f(u)$ satisfying the condition:

(A1) $|f(u)| \leq C|u|$, and $\left|f^{\prime}(u)\right| \leq C$.

It can be seen that the nonlinear term $r u(1-u / K)$ in Eq.(2.1) satisfies the condition (A1) due to the natural boundedness of the solution. In addition, the positive constant $C$ is arbitrary and can take different values throughout this paper if there is no specification.

\subsection{The Difference Scheme}

Denote

$$
\Omega_{h}=\left\{\left(x_{i}, t_{n}\right) \mid x_{i}=i h, i=0,1, \cdots, M ; t_{n}=n \tau, n=0,1, \cdots, N\right\},
$$

where $h=\frac{L}{M}$ is the space step, and $\tau=\frac{T}{N}$ is the time step. At the griding points Eq.(2.1) becomes

$$
\partial_{t}^{\alpha} u\left(x_{i}, t_{n+1}\right)=D u_{x x}\left(x_{i}, t_{n+1}\right)+f\left(x_{i}, t_{n+1}\right)+\gamma\left(x_{i}, t_{n+1}\right) .
$$


Firstly the Caputo's fractional derivative is discretized by

$$
\begin{aligned}
\partial_{t}^{\alpha} u\left(x_{i}, t_{n+1}\right) & =\frac{1}{\Gamma(1-\alpha)} \int_{0}^{t_{n+1}}\left(t_{n+1}-\eta\right)^{-\alpha} \frac{\partial u\left(x_{i}, \eta\right)}{\partial \eta} d \eta \\
& =\frac{\tau^{-\alpha}}{\Gamma(2-\alpha)} \sum_{k=0}^{n}\left(u\left(x_{i}, t_{n-k+1}\right)-u\left(x_{i}, t_{n-k}\right)\right)\left[(k+1)^{1-\alpha}-k^{\alpha}\right]+O(\tau) .
\end{aligned}
$$

Next the second-order derivative is discretized according to the general central difference

$$
\begin{aligned}
u_{x x}\left(x_{i}, t_{n+1}\right) & =\frac{1}{h^{2}}\left(u\left(x_{i-1}, t_{n+1}\right)-2 u\left(x_{i}, t_{n+1}\right)+u\left(x_{i+1}, t_{n+1}\right)\right)+O\left(h^{2}\right) \\
& :=\delta_{x}^{2} u\left(x_{i}, t_{n+1}\right)+O\left(h^{2}\right) .
\end{aligned}
$$

Finally for the nonlinear source term $f(u)$, there holds by Taylor's expansion

$$
f\left(u\left(x_{i}, t_{n+1}\right)\right)=2 f\left(u\left(x_{i}, t_{n}\right)\right)-f\left(u\left(x_{i}, t_{n-1}\right)\right)+O\left(\tau^{2}\right) .
$$

Substituting (2.6), (2.7) and (2.8) into (2.5), we have

Case I. $n=0$

$$
\frac{\tau^{-\alpha}}{\Gamma(2-\alpha)}\left[u\left(x_{i}, t_{1}\right)-u\left(x_{i}, t_{0}\right)\right]-D \delta_{x}^{2} u\left(x_{i}, t_{1}\right)=f\left(u\left(x_{i}, t_{0}\right)\right)+\gamma\left(x_{i}, t_{1}\right)+E_{0}+E_{1} .
$$

Case II. $n \geq 1$

$$
\begin{aligned}
& \frac{\tau^{-\alpha}}{\Gamma(2-\alpha)} \sum_{k=0}^{n}\left(u\left(x_{i}, t_{n-k+1}\right)-u\left(x_{i}, t_{n-k}\right)\right) c_{k}-D \delta_{x}^{2} u\left(x_{i}, t_{n+1}\right) \\
= & {\left[2 f\left(u\left(x_{i}, t_{n}\right)\right)-f\left(u\left(x_{i}, t_{n-1}\right)\right)\right]+\gamma\left(x_{i}, t_{n+1}\right)+E_{0}+E_{2} . }
\end{aligned}
$$

where

$$
c_{k}=(k+1)^{1-\alpha}-k^{1-\alpha}, k=0,1, \cdots,
$$

and

$$
\begin{aligned}
E_{0} & =O\left(\tau+h^{2}\right), \\
E_{1} & =f\left(u\left(x_{i}, t_{1}\right)\right)-f\left(u\left(x_{i}, t_{0}\right)\right)=f^{\prime}\left(u\left(x_{i}, t_{\xi}\right)\right)\left(u\left(x_{i}, t_{1}\right)-u\left(x_{i}, t_{0}\right)\right) \\
& =f^{\prime}\left(u\left(x_{i}, t_{\xi}\right)\right)\left(u_{t}\left(x_{i}, t_{\eta}\right) \tau\right), \\
E_{2} & =f\left(u\left(x_{i}, t_{n+1}\right)\right)-\left[2 f\left(u\left(x_{i}, t_{n}\right)\right)-f\left(u\left(x_{i}, t_{n-1}\right)\right)\right]=O\left(\tau^{2}\right) .
\end{aligned}
$$

Denote $u_{i}^{j} \approx u\left(x_{i}, t_{j}\right)$, and discretize the initial boundary values by

$$
u_{i}^{0}=u_{0}\left(x_{i}\right), u_{0}^{j}=u_{1}^{j}, u_{M}^{j}=u_{M-1}^{j} .
$$

Omitting the truncated terms, we get an implicit difference scheme given as

Case I. $n=0$

$$
\frac{\tau^{-\alpha}}{\Gamma(2-\alpha)}\left(u_{i}^{1}-u_{i}^{0}\right)-D \delta_{x}^{2} u_{i}^{1}=f\left(u_{i}^{0}\right)+\gamma_{i}^{1} .
$$

Case II. $n \geq 1$

$$
\frac{\tau^{-\alpha}}{\Gamma(2-\alpha)} \sum_{k=0}^{n}\left(u_{i}^{n-k+1}-u_{i}^{n-k}\right) c_{k}-D \delta_{x}^{2} u_{i}^{n+1}=\left[2 f\left(u_{i}^{n}\right)-f\left(u_{i}^{n-1}\right)\right]+\gamma_{i}^{n+1} .
$$

Denote

$$
\begin{gathered}
p=\frac{D \tau^{\alpha} \Gamma(2-\alpha)}{h^{2}}, q=\tau^{\alpha} \Gamma(2-\alpha), \\
U^{n}=\left(u_{1}^{n}, u_{2}^{n}, \cdots, u_{M-1}^{n}\right)^{T}, \\
\left(U^{n}\right)^{2}=\left(\left(u_{1}^{n}\right)^{2},\left(u_{2}^{n}\right)^{2}, \cdots,\left(u_{M-1}^{n}\right)^{2}\right), \\
G^{n}=\left(\gamma_{1}^{n}, \gamma_{2}^{n}, \cdots, \gamma_{M-1}^{n}\right) .
\end{gathered}
$$


Noting that $f(u)=r u-\frac{r}{K} u^{2}$, by rearrangement we get the difference scheme in matrix form:

$$
\left\{\begin{array}{l}
A U^{1}=(1+q r) U^{0}-\frac{q r}{K}\left(U^{0}\right)^{2}+q G^{1}, \\
A U^{n+1}=\sum_{j=1}^{n}\left(c_{n-1}-c_{n}\right) U^{n+1-j}+c_{n} U^{0}+2 q r U^{n}-\frac{2 q r}{K}\left(U^{n}\right)^{2}-q r U^{n-1}+\frac{q r}{K}\left(U^{n-1}\right)^{2}+q G^{n+1},
\end{array}\right.
$$

$n=1,2, \cdots, N-1$, where

$$
A=\left(a_{i j}\right)_{(M-1) \times(M-1)}=\left(\begin{array}{cccccc}
1+p & -p & 0 & \cdots & 0 & 0 \\
-p & 1+2 p & -p & \cdots & 0 & 0 \\
0 & -p & 1+2 p & \cdots & 0 & 0 \\
\vdots & \vdots & \vdots & \ddots & \vdots & \vdots \\
0 & 0 & 0 & \cdots & 1+2 p & -p \\
0 & 0 & 0 & \cdots & -p & 1+p
\end{array}\right)
$$

Noting to $p>0$, the coefficient matrix $A$ defined by (2.16) is strictly diagonal-dominant, then we have

Lemma 1 The difference scheme (2.15) is uniquely solvable.

Since $a_{i i}=1+\sum_{j=1, j \neq i}^{M-1}\left|a_{i j}\right|, i=1,2, \cdots, M-1$, we can not accurately estimate the spectral radius and obtain the stability and convergence of the difference scheme. We are to prove the stability and convergence based on the discrete forms (2.13)-(2.14). Therefore we need the discrete Gronwall inequality.

Lemma 2 Assume that $\left\{F_{k}, k \geq 0\right\}$ be nonnegative series and satisfy the condition:

$$
F_{k+1} \leq A+B s \sum_{l=0}^{k} F_{l}, k=0,1, \cdots,
$$

then there holds

$$
F_{k+1} \leq A \exp (B k s), k=0,1, \cdots
$$

where $A, B$ and $s$ are nonnegative constants.

In addition, for the coefficient $c_{k}$ given by (2.11), it is not difficult to testify

Lemma 3 The series $c_{k}, k=0,1, \cdots$, defined by (2.11), is strictly decreasing, and there holds

$$
0<c_{k}<c_{0}=1, k=1,2, \cdots ;
$$

and

$$
\sum_{n=1}^{N-1} c_{n}=N^{1-\alpha}-1, N \geq 2
$$

\section{Stability and Convergence}

In the follows, we denote $L^{2}$ inner by $(\cdot, \cdot)$, and $L^{2}$ norm by $\|\cdot\|$, and $C>0$ always represents a positive constant.

\subsection{Stability}

Theorem 1 For $0<T<\infty$ and $\tau$ small enough, there holds

$$
\Xi\left(u^{N}\right) \leq C\left(\frac{T^{1-\alpha}+\tau^{1-\alpha}}{2 \Gamma(2-\alpha)}+\tau\right)\left\|u_{0}\right\|^{2}+C \tau \sum_{n=1}^{N-1}\left\|\gamma^{n}\right\|^{2},
$$

and the finite difference scheme (2.15) is stable, where $\Xi\left(u^{n}\right)=\frac{\tau^{1-\alpha}}{2 \Gamma(2-\alpha)} \sum_{k=0}^{n-1} c_{k}\left\|u^{n-k}\right\|^{2}$, and $C$ is an arbitrary positive constant but independent of $h$ and $\tau$.

Proof Firstly consider the discrete equation (2.14). By $u_{i}^{n+1}$ making inner product with all terms in (2.14) respectively, and summing on $i(i=1,2, \cdots, M-1)$, there holds

$$
\begin{aligned}
& \left(\frac{\tau^{-\alpha}}{\Gamma(2-\alpha)} \sum_{k=0}^{n}\left(u^{n-k+1}-u^{n-k}\right) c_{k}, u^{n+1}\right)-\left(D \delta_{x}^{2} u^{n+1}, u^{n+1}\right) \\
= & \left(2 f\left(u^{n}\right)-f\left(u^{n-1}\right), u^{n+1}\right)+\left(\gamma^{n+1}, u^{n+1}\right) .
\end{aligned}
$$


Noting that

$$
\begin{aligned}
& \left(\frac{\tau^{-\alpha}}{\Gamma(2-\alpha)} \sum_{k=0}^{n}\left(u^{n-k+1}-u^{n-k}\right) c_{k}, u^{n+1}\right) \\
= & \frac{\tau^{-\alpha}}{\Gamma(2-\alpha)}\left(c_{0} u^{n+1}-\sum_{k=0}^{n-1}\left(c_{k}-c_{k+1}\right) u^{n-k}-c_{n} u^{0}, u^{n+1}\right) \\
= & \frac{\tau^{-\alpha}}{\Gamma(2-\alpha)}\left(c_{0}\left\|u^{n+1}\right\|^{2}-\sum_{k=0}^{n-1}\left(c_{k}-c_{k+1}\right)\left(u^{n-k}, u^{n+1}\right)-c_{n}\left(u^{0}, u^{n+1}\right)\right),
\end{aligned}
$$

and

$$
\left(D \delta_{x}^{2} u^{n+1}, u^{n+1}\right)=-D\left(\delta_{x} u^{n+1}, \delta_{x} u^{n+1}\right)=-D\left\|\delta_{x} u^{n+1}\right\|^{2},
$$

we have

$$
\begin{aligned}
& \frac{\tau^{-\alpha}}{\Gamma(2-\alpha)}\left(c_{0}\left\|u^{n+1}\right\|^{2}-\sum_{k=0}^{n-1}\left(c_{k}-c_{k+1}\right)\left(u^{n-k}, u^{n+1}\right)-c_{n}\left(u^{0}, u^{n+1}\right)\right)+D\left\|\delta_{x} u^{n+1}\right\|^{2} \\
= & \left(2 f\left(u^{n}\right)-f\left(u^{n-1}\right), u^{n+1}\right)+\left(\gamma^{n+1}, u^{n+1}\right) .
\end{aligned}
$$

Thanks to the nonnegativity of $D\left\|\delta_{x} u^{n+1}\right\|^{2}$, we get

$$
\begin{aligned}
& \frac{\tau^{-\alpha}}{\Gamma(2-\alpha)}\left(c_{0}\left\|u^{n+1}\right\|^{2}-\sum_{k=0}^{n}\left(c_{k}-c_{k+1}\right)\left(u^{n-k}, u^{n+1}\right)-c_{n}\left(u^{0}, u^{n+1}\right)\right) \\
\leq & \left(2 f\left(u^{n}\right)-f\left(u^{n-1}\right), u^{n+1}\right)+\left(\gamma^{n+1}, u^{n+1}\right) .
\end{aligned}
$$

Noting $c_{0}=1$, there holds by Cauchy-Schwarz inequality

$$
\begin{aligned}
& \frac{\tau^{-\alpha}}{\Gamma(2-\alpha)}\left\|u^{n+1}\right\|^{2} \\
\leq & \frac{\tau^{-\alpha}}{2 \Gamma(2-\alpha)}\left[\sum_{k=0}^{n-1}\left(c_{k}-c_{k+1}\right)\left(\left\|u^{n-k}\right\|^{2}+\left\|u^{n+1}\right\|^{2}\right)+c_{n}\left(\left\|u^{0}\right\|^{2}+\left\|u^{n+1}\right\|^{2}\right)\right] \\
& +\left(2 f\left(u^{n}\right)-f\left(u^{n-1}\right), u^{n+1}\right)+\left(\gamma^{n+1}, u^{n+1}\right) \\
= & \frac{\tau^{-\alpha}}{2 \Gamma(2-\alpha)}\left[\sum_{k=0}^{n-1}\left(c_{k}-c_{k+1}\right)\left\|u^{n-k}\right\|^{2}+\left\|u^{n+1}\right\|^{2}+c_{n}\left\|u^{0}\right\|^{2}\right] \\
& +\left(2 f\left(u^{n}\right)-f\left(u^{n-1}\right), u^{n+1}\right)+\left(\gamma^{n+1}, u^{n+1}\right) .
\end{aligned}
$$

That is

$$
\begin{aligned}
& \frac{\tau^{-\alpha}}{2 \Gamma(2-\alpha)}\left\|u^{n+1}\right\|^{2}+\frac{\tau^{-\alpha}}{2 \Gamma(2-\alpha)} \sum_{k=0}^{n-1} c_{k+1}\left\|u^{n-k}\right\|^{2} \\
\leq & \frac{\tau^{-\alpha}}{2 \Gamma(2-\alpha)} \sum_{k=0}^{n-1} c_{k}\left\|u^{n-k}\right\|^{2}+\frac{\tau^{-\alpha}}{2 \Gamma(2-\alpha)} c_{n}\left\|u^{0}\right\|^{2}+\left(2 f\left(u^{n}\right)-f\left(u^{n-1}\right), u^{n+1}\right)+\left(\gamma^{n+1}, u^{n+1}\right) .
\end{aligned}
$$

Since

$$
\frac{\tau^{-\alpha}}{2 \Gamma(2-\alpha)}\left\|u^{n+1}\right\|^{2}+\frac{\tau^{-\alpha}}{2 \Gamma(2-\alpha)} \sum_{k=0}^{n-1} c_{k+1}\left\|u^{n-k}\right\|^{2}=\frac{\tau^{-\alpha}}{2 \Gamma(2-\alpha)} \sum_{k=0}^{n} c_{k}\left\|u^{n+1-k}\right\|^{2},
$$

by $\tau>0$ multiplying the two-sides of (3.7), there holds

$$
\begin{aligned}
& \frac{\tau^{1-\alpha}}{2 \Gamma(2-\alpha)} \sum_{k=0}^{n} c_{k}\left\|u^{n+1-k}\right\|^{2} \\
\leq & \frac{\tau^{1-\alpha}}{2 \Gamma(2-\alpha)} \sum_{k=0}^{n-1} c_{k}\left\|u^{n-k}\right\|^{2}+\frac{\tau^{1-\alpha}}{2 \Gamma(2-\alpha)} c_{n}\left\|u^{0}\right\|^{2}+\tau\left(2 f\left(u^{n}\right)-f\left(u^{n-1}\right), u^{n+1}\right)+\tau\left(\gamma^{n+1}, u^{n+1}\right) .
\end{aligned}
$$

Denote $\Xi\left(u^{n}\right)=\frac{\tau^{1-\alpha}}{2 \Gamma(2-\alpha)} \sum_{k=0}^{n-1} c_{k}\left\|u^{n-k}\right\|^{2}$. Noting the condition $|f(u)| \leq C|u|$, we have by using the Cauchy-Schwarz inequality again

$$
\begin{aligned}
& \tau\left(2 f\left(u^{n}\right)-f\left(u^{n-1}\right), u^{n+1}\right)+\tau\left(\gamma^{n+1}, u^{n+1}\right) \\
\leq & C \tau\left(\left\|u^{n}\right\|^{2}+\left\|u^{n+1}\right\|^{2}+\left\|u^{n-1}\right\|^{2}+\left\|\gamma^{n+1}\right\|^{2}\right) .
\end{aligned}
$$


Substituting (3.9) into (3.8), there holds

$$
\Xi\left(u^{n+1}\right) \leq \Xi\left(u^{n}\right)+\frac{\tau^{1-\alpha}}{2 \Gamma(2-\alpha)} c_{n}\left\|u^{0}\right\|^{2}+C \tau\left(\left\|u^{n}\right\|^{2}+\left\|u^{n+1}\right\|^{2}+\left\|u^{n-1}\right\|^{2}+\left\|\gamma^{n+1}\right\|^{2}\right),
$$

and summing on $n$ form $n=1$ to $n=N-1$, we get

$$
\Xi\left(u^{N}\right) \leq \Xi\left(u^{1}\right)+\frac{\tau^{1-\alpha}}{2 \Gamma(2-\alpha)} \sum_{n=1}^{N-1} c_{n}\left\|u^{0}\right\|^{2}+C \tau \sum_{n=1}^{N-1}\left(\left\|u^{n}\right\|^{2}+\left\|u^{n+1}\right\|^{2}+\left\|u^{n-1}\right\|^{2}+\left\|\gamma^{n+1}\right\|^{2}\right) .
$$

Thanks to $\left\|u^{n}\right\|^{2} \leq C \Xi\left(u^{n}\right)$ and $\tau N \leq T$, we have by Lemma 3

$$
\begin{aligned}
& (1-C \tau) \Xi\left(u^{N}\right) \\
& \leq \Xi\left(u^{1}\right)+\left(\frac{\tau^{1-\alpha}}{2 \Gamma(2-\alpha)} \sum_{n=1}^{N-1} c_{n}+C \tau\right)\left\|u^{0}\right\|^{2}+C \tau \sum_{n=1}^{N-1} \Xi\left(u^{n}\right)+C \tau \sum_{n=1}^{N-1}\left\|\gamma^{n+1}\right\|^{2} \\
& \leq \Xi\left(u^{1}\right)+\left[\frac{\tau^{1-\alpha}}{2 \Gamma(2-\alpha)}\left(N^{1-\alpha}-1\right)+C \tau\right]\left\|u^{0}\right\|^{2}+C \tau \sum_{n=1}^{N-1} \Xi\left(u^{n}\right)+C \tau \sum_{n=1}^{N-1}\left\|\gamma^{n+1}\right\|^{2} \\
& \leq \Xi\left(u^{1}\right)+\left(\frac{T^{1-\alpha}}{2 \Gamma(2-\alpha)}+C \tau\right)\left\|u^{0}\right\|^{2}+C \tau \sum_{n=1}^{N-1} \Xi\left(u^{n}\right)+C \tau \sum_{n=1}^{N-1}\left\|\gamma^{n+1}\right\|^{2} .
\end{aligned}
$$

Let the time step $\tau$ small enough such that $1-C \tau>0$. Noting that $n \tau \leq T$ for $n=1, \cdots, N$, we have by using Lemma 2

$$
\Xi\left(u^{N}\right) \leq C \Xi\left(u^{1}\right)+C\left(\frac{T^{1-\alpha}}{2 \Gamma(2-\alpha)}+\tau\right)\left\|u^{0}\right\|^{2}+C \tau \sum_{n=1}^{N-1}\left\|\gamma^{n+1}\right\|^{2} .
$$

Based on (2.13), with a completely similar method as used in the above to estimate $\Xi\left(u^{1}\right)$, there holds

$$
\Xi\left(u^{1}\right) \leq C\left(\frac{\tau^{1-\alpha}}{2 \Gamma(2-\alpha)}+\tau\right)\left\|u^{0}\right\|^{2}+C \tau\left\|\gamma^{1}\right\|^{2},
$$

by substituting it into (3.13) follows that (3.1) is valid. The proof is over.

\subsection{Convergence}

Theorem 2 The difference scheme (2.15) is convergent as $h, \tau \rightarrow 0$ for given $T<+\infty$, and there holds

$$
\left\|e^{n}\right\|_{2}^{2} \leq C \tau^{\alpha}\left(\tau^{2}+h^{4}\right)
$$

where $e^{n}=\left(e_{1}^{n}, \cdots, e_{M}^{n}\right)^{T}$ denotes the solutions error, and

$$
e_{i}^{n}=u\left(x_{i}, t^{n}\right)-u_{i}^{n}, i=1, \cdots, M ; n=1, \cdots, N .
$$

Proof Also consider the discrete scheme (2.13)-(2.14). By (2.13) subtracting (2.9) and (2.14) subtracting (2.10), respectively, there holds

Case I. $n=0$

$$
\frac{\tau^{-\alpha}}{\Gamma(2-\alpha)}\left(e_{i}^{1}-e_{i}^{0}\right)-D \delta_{x}^{2} e_{i}^{1}=E_{1}+E_{0}
$$

Case II. $n \geq 1$

$$
\frac{\tau^{-\alpha}}{\Gamma(2-\alpha)} \sum_{k=0}^{n}\left(e_{i}^{n-k+1}-e_{i}^{n-k}\right) c_{k}-D \delta_{x}^{2} e_{i}^{n+1}=\varepsilon_{n}+E_{2}+E_{0},
$$

where $E_{0}, E_{1}$ and $E_{2}$ are given by (2.12), and

$$
\varepsilon_{n}=2 f\left(u\left(x_{i}, t^{n}\right)\right)-f\left(u\left(x_{i}, t_{n-1}\right)\right)-2 f\left(u_{i}^{n}\right)+f\left(u_{i}^{n-1}\right) .
$$

By $e_{i}^{n+1}$ making inner product with the terms of (3.17), and summing on $i$ for $i=1,2, \cdots, M-1$, we have

$$
\begin{aligned}
& \frac{\tau^{-\alpha}}{\Gamma(2-\alpha)}\left(\sum_{k=0}^{n}\left(e^{n-k+1}-e^{n-k}\right) c_{k}, e^{n+1}\right)-D\left(\delta_{x}^{2} e^{n+1}, e^{n+1}\right) \\
= & \left(\varepsilon_{n}, e^{n+1}\right)+\left(E_{2}, e^{n+1}\right)+\left(E_{0}, e^{n+1}\right) .
\end{aligned}
$$


With a similar method as used in the proof of stability, we get

$$
\begin{aligned}
& \frac{\tau^{1-\alpha}}{2 \Gamma(2-\alpha)} \sum_{k=0}^{n} c_{k}\left\|e^{n+1-k}\right\|^{2} \\
\leq & \frac{\tau^{1-\alpha}}{2 \Gamma(2-\alpha)} \sum_{k=0}^{n-1} c_{k}\left\|e^{n-k}\right\|^{2}+\frac{\tau^{1-\alpha}}{2 \Gamma(2-\alpha)} c_{n}\left\|e^{0}\right\|^{2} \\
+ & \tau\left(\varepsilon_{n}, e^{n+1}\right)+\tau\left(E_{2}, e^{n+1}\right)+\tau\left(E_{0}, e^{n+1}\right) .
\end{aligned}
$$

Noting the definition of $\Xi$, there holds

$$
\Xi\left(e^{n+1}\right) \leq \Xi\left(e^{n}\right)+\frac{\tau^{1-\alpha}}{2 \Gamma(2-\alpha)} c_{n}\left\|e^{0}\right\|^{2}+\tau\left(\varepsilon_{n}, e^{n+1}\right)+\tau\left(E_{2}, e^{n+1}\right)+\tau\left(E_{0}, e^{n+1}\right) .
$$

By the condition of $\left|f^{\prime}(u)\right| \leq C$ and utilizing the triangular inequality, we have

$$
\begin{aligned}
& \left(\varepsilon_{n}, e^{n+1}\right) \\
= & \left(2 f\left(u\left(x_{i}, t_{n}\right)\right)-f\left(u\left(x_{i}, t_{n-1}\right)\right)-2 f\left(u_{i}^{n}\right)+f\left(u_{i}^{n-1}\right), e^{n+1}\right) \\
\leq & C\left\|2 f^{\prime}\left(v^{n}\right) e^{n}-f^{\prime}\left(v^{n-1}\right) e^{n-1}\right\|^{2}+C\left\|e^{n+1}\right\|^{2} \\
\leq & C\left(\left\|e^{n}\right\|^{2}+\left\|e^{n-1}\right\|^{2}+\left\|e^{n+1}\right\|^{2}\right) .
\end{aligned}
$$

Thus (3.21) reduces to

$$
\begin{aligned}
\Xi\left(e^{n+1}\right) & \leq \Xi\left(e^{n}\right)+\frac{\tau^{1-\alpha}}{2 \Gamma(2-\alpha)} c_{n}\left\|e^{0}\right\|^{2}+C \tau\left(\left\|e^{n}\right\|^{2}+\left\|e^{n-1}\right\|^{2}+\left\|e^{n+1}\right\|^{2}\right) \\
& +C \tau\left(\left\|E_{0}\right\|^{2}+\left\|E_{2}\right\|^{2}\right) .
\end{aligned}
$$

Making summation on $n$ for $n=1, \cdots, N-1$, we get

$$
\begin{aligned}
\Xi\left(e^{N}\right) & \leq \Xi\left(e^{1}\right)+\frac{\tau^{1-\alpha}}{2 \Gamma(2-\alpha)} \sum_{n=1}^{N-1} c_{n}\left\|e^{0}\right\|^{2}+C \tau \sum_{n=1}^{N-1}\left(\left\|e^{n}\right\|^{2}+\left\|e^{n-1}\right\|^{2}+\left\|e^{n+1}\right\|^{2}\right) \\
& +C \tau\left(\left\|E_{0}\right\|^{2}+\left\|E_{2}\right\|^{2}\right) .
\end{aligned}
$$

Noting that $e_{i}^{0}=0$ and the arbitrary property of the constant $C>0$, we have by using the estimates of $E_{0}$ and $E_{2}$

$$
\Xi\left(e^{N}\right) \leq C \Xi\left(e^{1}\right)+C \tau \sum_{n=1}^{N-1} \Xi\left(e^{n}\right)+C \tau\left(\tau^{2}+h^{4}\right) .
$$

Similarly, by $e_{i}^{1}$ making the inner product with the terms of (3.16), and summing on $i(i=1, \cdots, M-1)$, there holds

$$
\Xi\left(e^{1}\right) \leq C \tau\left(\tau^{2}+h^{4}\right),
$$

and substituting it into (3.25), and utilizing Lemma 2 again we get

$$
\Xi\left(e^{N}\right) \leq C \tau\left(\tau^{2}+h^{4}\right) .
$$

Noting the definition of $\Xi\left(e^{N}\right)$ there holds

$$
\frac{\tau^{1-\alpha}}{2 \Gamma(2-\alpha)} \sum_{k=0}^{N-1} c_{k}\left\|e^{N-k}\right\|^{2} \leq C \tau\left(\tau^{2}+h^{4}\right), \quad N \geq 2,
$$

by the arbitrariness of $C>0$ again implies that the assertion (3.15) is valid. The proof is completed.

\section{Numerical Tests}

\subsection{Example 4.1}

Without loss of generality, we take the fractional order $\alpha=0.5$ as example, and the model parameters are chosen as $D=0.001, r=0.01$ and $L=1, K=10$. Let the exact solution of the forward problem be

$$
u(x, t)=x^{2}(1-x)^{2}(1-t)
$$


and the force term is

$$
\gamma(x, t)=-\frac{x^{2}(1-x)^{2} t^{1-\alpha}}{\Gamma(2-\alpha)}-2 D(1-t)\left(6 x^{2}-6 x+1\right)-r x^{2}(1-x)^{2}(1-t)+\frac{r}{K} x^{4}(1-x)^{4}(1-t)^{2} .
$$

The relative error in the exact and numerical solutions at $t=0.5$ is expressed by

$$
\operatorname{Err}=\frac{\left\|u(x, 0.5)-u^{*}(x, 0.5)\right\|_{2}}{\|u(x, 0.5)\|_{2}} .
$$

By using the finite difference scheme (2.15) to solve the problem, and the computational results are listed in Table 1, Table 2 with fixed time step and space step respectively. From Table 1 and Table 2 it can be found that the solutions errors become small as the space-time steps going to small, but the convergent rate is slow relatively.

By Theorem 2, we recompute the problem by taking $\tau=h^{2}$, and other parameters unchanged. The computational results are listed in Table 3, where Rat denotes the convergent rate. From Table 3 it can be found that the numerical solutions converge to the exact solution almost with the order of $2+\alpha$ as the grids becoming fine, which basically coincides with the theoretical analysis in Theorem 2. Furthermore, also taking $\alpha=0.5$, the exact solution and the numerical solutions at $t=0.5$, as $\tau=h^{2}$ with $h=1 / 10,1 / 20,1 / 40$ and $h=1 / 80$, are plotted in Figure 1. It can be seen clearly that the numerical solutions converge to the exact solution as the grids becoming fine.

Table 1. The solutions errors with space $\operatorname{steps}(\tau=1 / 100)$

\begin{tabular}{lccccc}
\hline$h$ & $1 / 20$ & $1 / 40$ & $1 / 60$ & $1 / 80$ & $1 / 100$ \\
\hline Err & $2.53697 \mathrm{e}-2$ & $8.24423 \mathrm{e}-3$ & $5.11389 \mathrm{e}-3$ & $4.01149 \mathrm{e}-3$ & $3.504 \mathrm{e}-3$ \\
\hline
\end{tabular}

Table 2. The solutions errors with time steps $(h=1 / 100)$

\begin{tabular}{lccccc}
\hline$\tau$ & $1 / 20$ & $1 / 40$ & $1 / 60$ & $1 / 80$ & $1 / 100$ \\
\hline Err & $3.19053 \mathrm{e}-2$ & $1.09364 \mathrm{e}-2$ & $6.20061 \mathrm{e}-3$ & $4.37592 \mathrm{e}-3$ & $3.504 \mathrm{e}-3$ \\
\hline
\end{tabular}

Table 3. The solutions errors with time-space steps in Ex.4.1

\begin{tabular}{lccc}
\hline$h$ & $\tau=h^{2}$ & Err & Rat \\
\hline $1 / 10$ & $1 / 100$ & $1.00646 \mathrm{e}-1$ & \\
$1 / 20$ & $1 / 400$ & $2.49254 \mathrm{e}-2$ & 4.0379 \\
$1 / 40$ & $1 / 1600$ & $7.55186 \mathrm{e}-3$ & 3.3006 \\
$1 / 80$ & $1 / 6400$ & $2.79854 \mathrm{e}-3$ & 2.6985 \\
$1 / 160$ & $1 / 25600$ & $1.18621 \mathrm{e}-3$ & 2.3592 \\
\hline
\end{tabular}

\subsection{Example 4.2}

Let the exact solution of the forward problem be

$$
u(x, t)=e^{\frac{1}{2} x^{2}-\frac{1}{3} x^{3}} t^{2} .
$$

The other parameters are the same as in Ex.4.1, and the force term here is

$$
\begin{aligned}
& \gamma(x, t)=\frac{2 e^{\frac{1}{2} x^{2}-\frac{1}{3} x^{3}} t^{2-\alpha}}{\Gamma(3-\alpha)}-D e^{\frac{1}{2} x^{2}-\frac{1}{3} x^{3}}\left[x^{2}(1-x)^{2}+1-2 x\right] t^{2} \\
& -r e^{\frac{1}{2} x^{2}-\frac{1}{3} x^{3}} t^{2}+\frac{r}{K} e^{x^{2}-\frac{2}{3} x^{3}} t^{4} .
\end{aligned}
$$

As done in Ex.4.1, the problem is solved by the finite difference scheme (2.15) by taking the grids of $\tau=h^{2}$, and the computational results are given in Table 4. It can be found that the numerical solutions approximate to the exact solution as the grids going to fine with a similar rate as that in Ex.4.1. Moreover, the exact and numerical solutions with different grids also at $t=0.5$ are plotted in Figure 2, and it can be seen again that the numerical solutions converge to the exact solution.

Table 4. The solutions errors with time-space steps in Ex.4.2

\begin{tabular}{lccc}
\hline$h$ & $\tau=h^{2}$ & Err & Rat \\
\hline $1 / 10$ & $1 / 100$ & $2.43968 \mathrm{e}-3$ & \\
$1 / 20$ & $1 / 400$ & $4.61353 \mathrm{e}-4$ & 5.2881 \\
$1 / 40$ & $1 / 1600$ & $1.07166 \mathrm{e}-4$ & 4.3050 \\
$1 / 80$ & $1 / 6400$ & $3.28737 \mathrm{e}-5$ & 3.2599 \\
$1 / 160$ & $1 / 25600$ & $1.25486 \mathrm{e}-5$ & 2.6197 \\
\hline
\end{tabular}




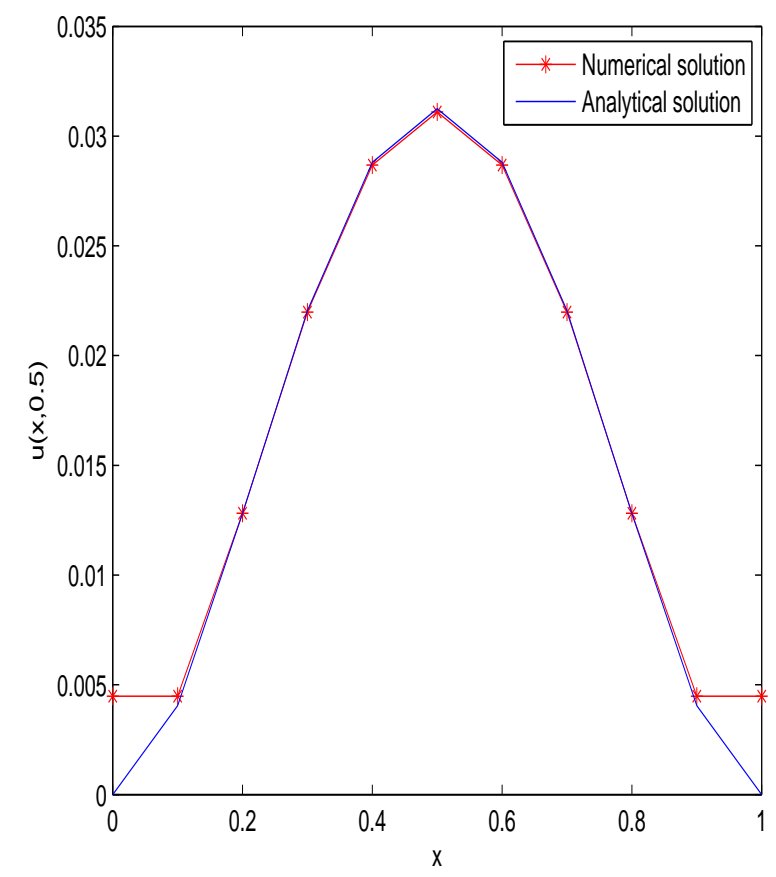

(a) $h=1 / 10$

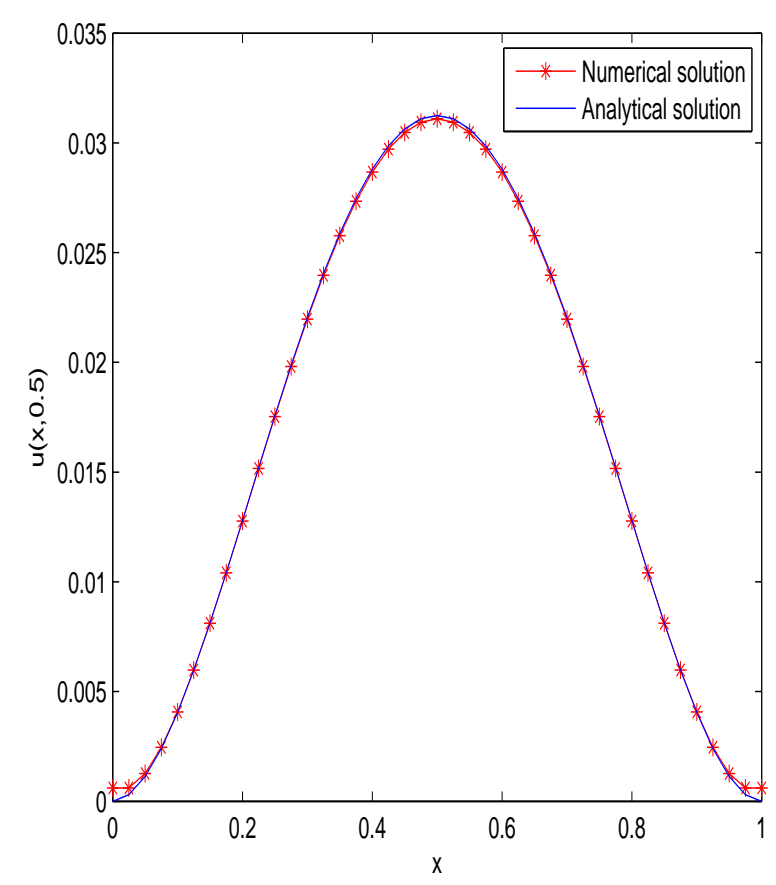

(c) $h=1 / 40$

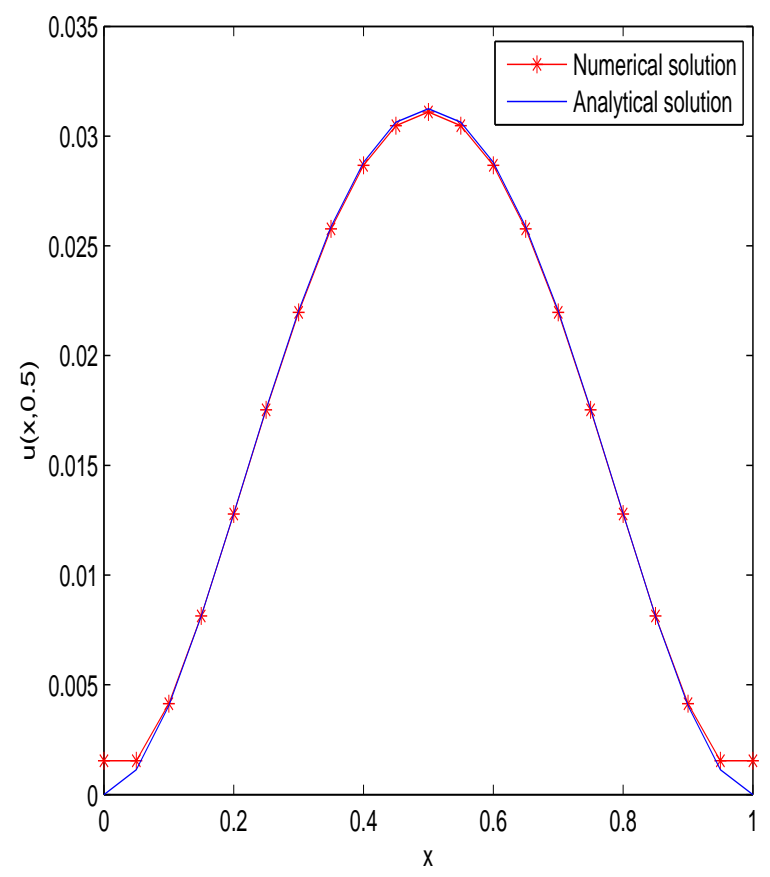

(b) $h=1 / 20$

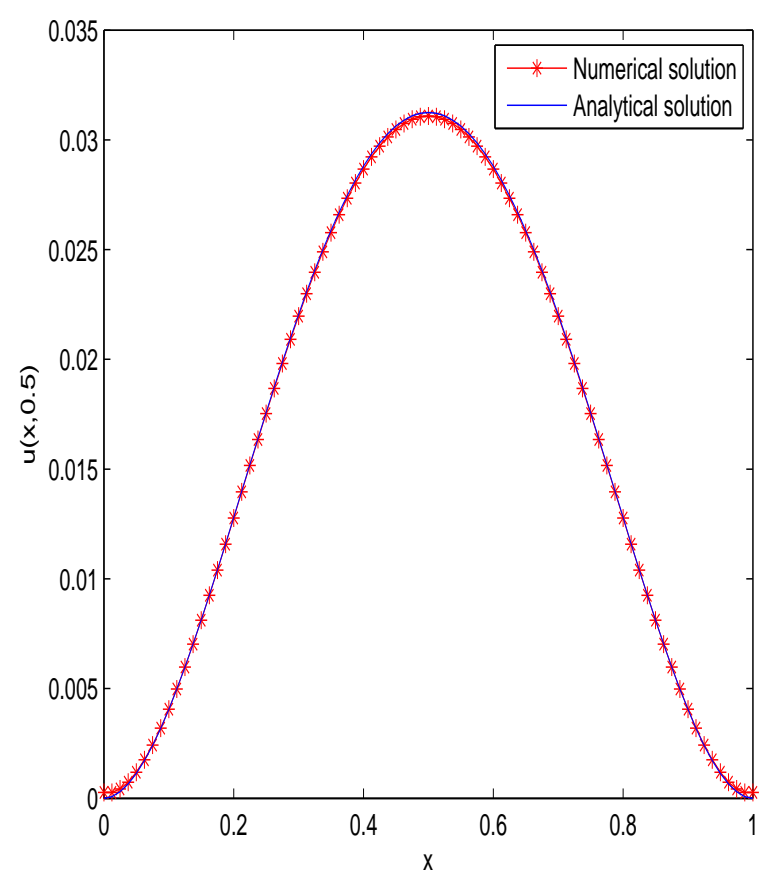

(d) $h=1 / 80$

Figure 1. The exact and numerical solutions in Ex.4.1 


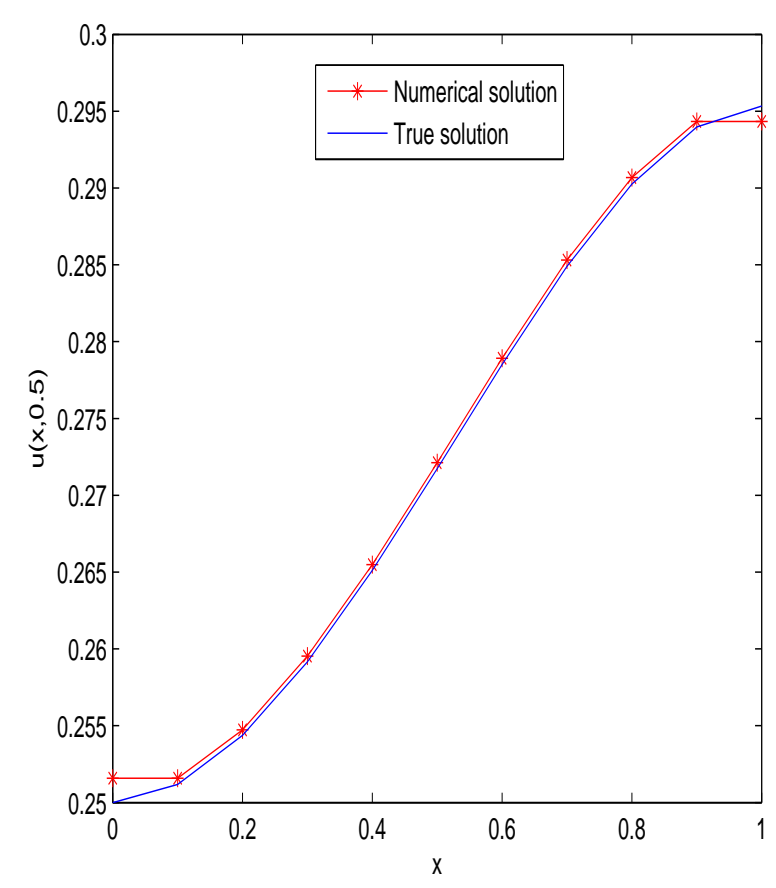

(a) $h=1 / 10$

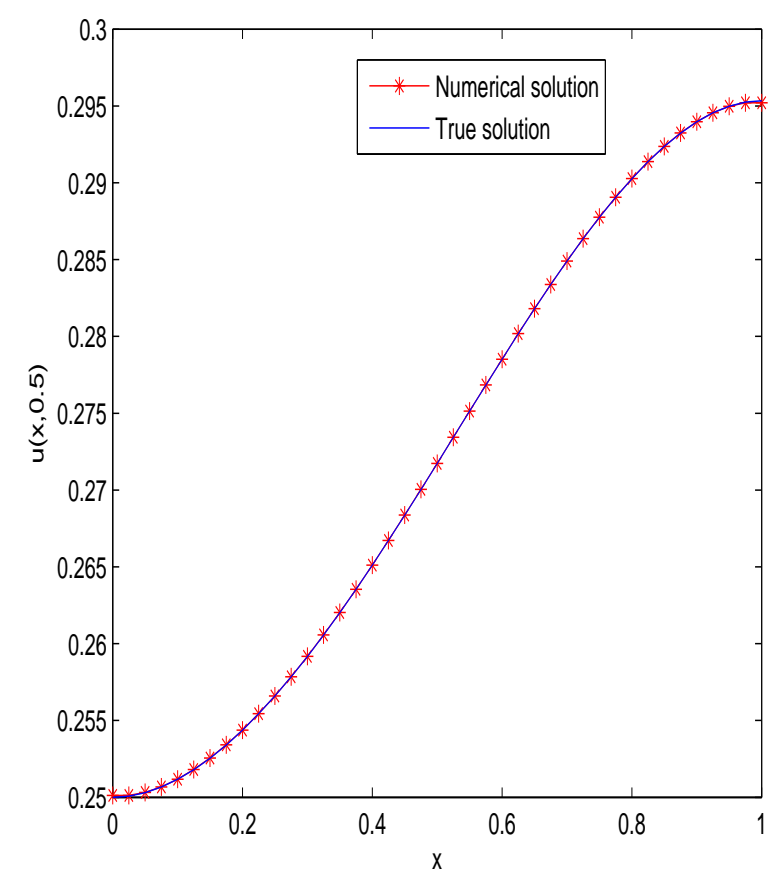

(c) $h=1 / 40$

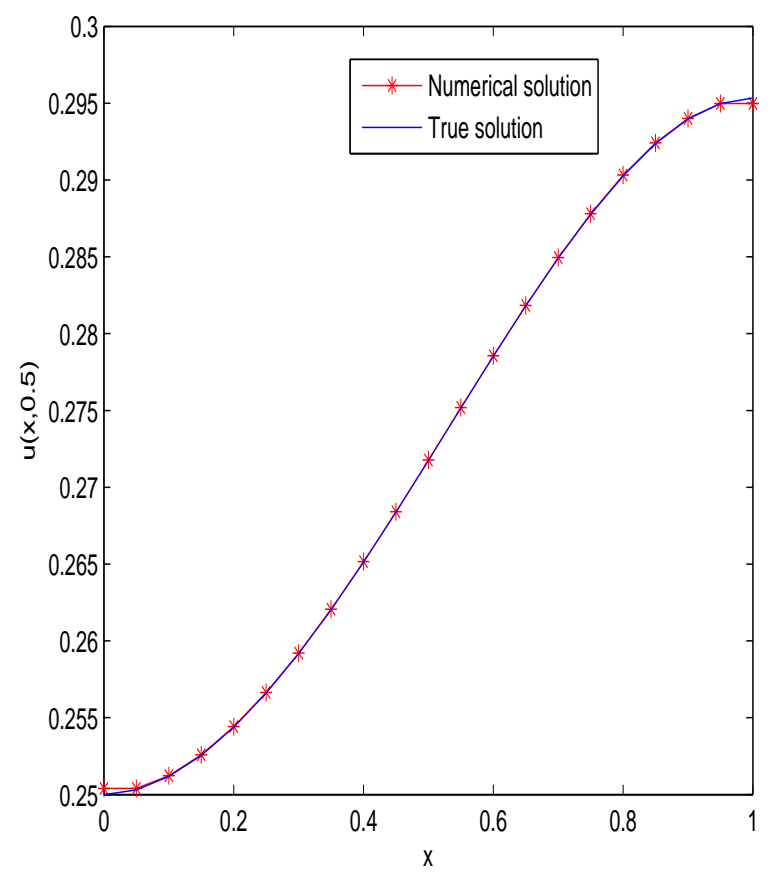

(b) $h=1 / 20$

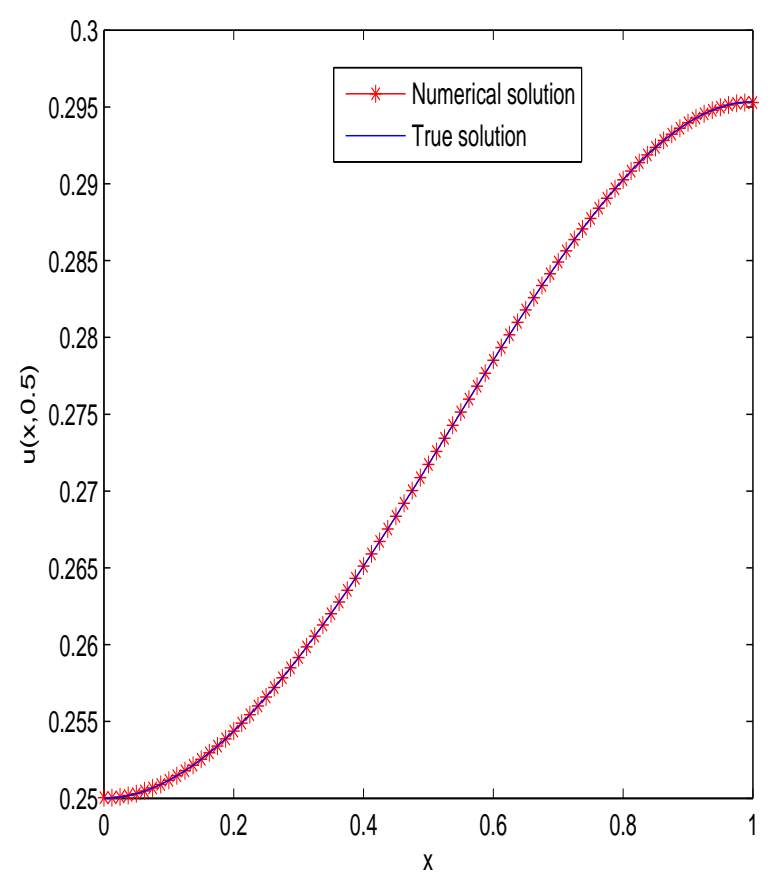

(d) $h=1 / 80$

Figure 2. The exact and numerical solutions in Ex.4.2 


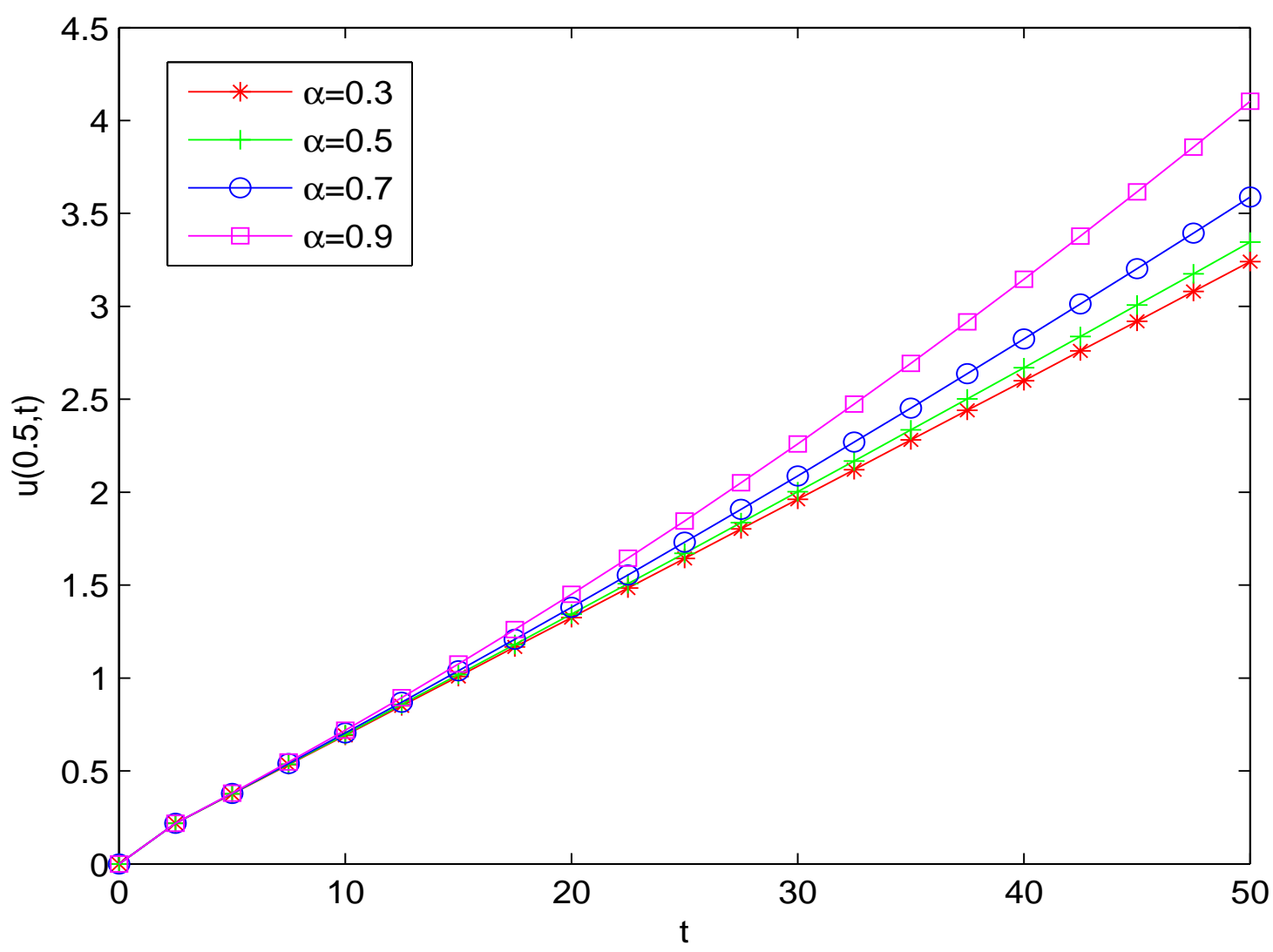

Figure 3. Long time behaviors of numerical solutions with fractional orders

\section{Remark 1}

The fractional order plays an important role in the fractional logistic diffusion model, especially for long time behaviors of the solution. Without loss of generality, let the exact solution of the forward problem be

$$
u(x, t)=x^{2}(1-x)^{2}(1+t),
$$

and the force term is

$$
\gamma(x, t)=\frac{x^{2}(1-x)^{2} t^{1-\alpha}}{\Gamma(2-\alpha)}-2 D\left(6 x^{2}-6 x+1\right)(1+t)-r x^{2}(1-x)^{2}(1+t)+\frac{r}{K} x^{4}(1-x)^{4}(1+t)^{2},
$$

and the model parameters are chosen as $D=0.01, r=0.01$ and $L=1, K=10$. By using the difference scheme (2.15), and taking $T=50$, the numerical solutions at $x=0.5$ with the time variables and different fractional orders are plotted in Figure 3. From Figure 3 it can be seen that the fractional orders have impacts on the growth behavior of the solution of the fractional logistic model with long time diffusion. The small of the fractional order, the slow of growth behavior of the solution after $t \geq 20$. However, when $t<10$, the solutions almost have the same behaviors even with different fractional orders.

\section{Conclusion}

A logistic-type model with space diffusion using fractional derivative is discussed which is of important meaning in spatial ecology. Such model is nonlinear and nonlocal which is unable to get its analytical solution. An implicit finite difference scheme is established and applied to give its numerical solution which is testified to be efficiency by numerical experiments, and long time behaviors of the solution with different fractional orders are observed. We will focus on regularity of solution of the forward problem and pay attention to inverse coefficient problems on the fractional logistic model. 


\section{Acknowledgements}

The authors thank the anonymous referees and the editor for their valuable and suggestive comments. This work is supported by National Natural Science Foundation of China (no. 11871313), and Natural Science Foundation of Shandong Province, China (no. ZR2019MA021).

\section{References}

Bai, L., \& Wang, K. (2005). Giplin-Ayala model with spatial diffusion and its optimal harvesting policy. Appl. Math. Comput., 171, 531-546. https://doi.org/10.1016/j.amc.2005.01.068

Balanov, L., Krawcewicz, W., \& Ruan, H. (2008). Hutchinson's delay logistic system with symmetries and spatial diffusion. Nonlinear Anal. Real World Appl., 9, 154-182.https://doi.org/10.1016/j.nonrwa.2006.09.013

Brauer, F., \& Castillo-Chavez, C. (2012). Mathematical Models in Population Biology and Epidemiology (Second Edition). New York: Springer. https://doi.org/10.1007/978-1-4614-1686-9

Chakraborty, A., Singh, M., Lucy, D., \& Ridland, P. (2007). Predator-prey model with prey-taxis and diffusion. Math. Comput. Modelling, 46, 482-498. https://doi.org/10.1016/j.mcm.2006.10.010

Chen, W., Sun, H. G., \& Li, X. C. (2010). Fractional Derivatives Modelling in Mechanics and Engineering (in Chinese). Beijing: Science Press.

Dawson, C. N., Wheeler, M. F., \& Woodward, C. S. (1998). A two-grid finite difference scheme for nonlinear parabolic equations. SIAM J. Numer. Anal., 35, 435-452. https://doi.org/10.1137/S0036142995293493

El-Nabulsi, R. A. (2011). The fractional Boltzmann transport equation. Computers and Mathematics with Applications, 62, 1568-1575. https://doi.org/10.1016/j.camwa.2011.03.040

El-Nabulsi, R. A. (2019). Fractional Navier-Stokes equation from fractional velocity arguments and its implications in fluid and microfilaments. International Journal of Nonlinear Sciences and Numerical Simulation, 20, 449-459. https://doi.org/10.1515/ijnsns-2018-0253

El-Nabulsi, R. A. (2020). On a new fractional uncertainty relation and its implications in quantum mechanics and molecular physics. Proc. Royal Soc. A, 476, 20190729. https://doi.org/10.1098/rspa.2019.0729

Jin, Y., \& Zhao, X. Q. (2008). Bistable waves for a class of cooperative reaction-diffusion systems. J. Biol. Dynamics, 2, 196-207. https://doi.org/10.1080/17513750802504823

Kelly, J. F., \& Meeschaert, M. M. (2019). Space-time duality and high-order fractional diffusion. Phys. Rev. E, 99, 022122. https://doi.org/10.1103/PhysRevE.99.022122

Korobenko, L., \& Braverman, E. (2009). A logistic model with a carrying capacity driven diffusion. Can. Appl. Math. Q., 17, 85-104.

Li, X. L., \& Rui, H. X. (2017). A two-grid block-centered finite difference method for the nonlinear time-fractional parabolic equation. J. Sci. Comput., 72, 863-891. https://doi.org/10.1007/s10915-017-0380-4

Lima, F. M. M., Machado, J. A. T., \& Crisostomo, M. (2007). Experimental signal analysis of Robot impacts in a fractional calculus perspective. J. Advanced Computational Intelligence and Intelligent Informatics, 11, 1079-1085.

Liu, Y., Du, Y. W., Li, H., He, S., \& Gao, W. (2015). Finite difference/finite element method for a nonlinear time-fractional fourth-order reaction-diffusion problem. Comput. Math. Appl., 35, 435-452. https://doi.org/10.1016/j.camwa.2015.05.015

Liu, Y., Du, Y. W., Li, H., \& Wang, J. F. (2016). A two-grid finite element approximation for a nonlinear time-fractional Cable equation. Nonlinear Dyn., 85, 2535-2548. https://doi.org/10.1007/s11071-016-2843-9

Liu, Y., Yu, Z. D., Li, H., Liu, F. W., \& Wang, J. F. (2018). Time two-mesh algorithm combined with finite element method for time-fractional water wave model. Inter. J. Heat Mass Transfer, 120, 1132-1145.

Luchko, Y., Rundell, W., Yamamoto, M., \& Zuo, L. H. (2013). Uniqueness and reconstruction of an unknown semilinear term in a time-fractional reactionCdiffusion equation. Inverse Problems, 29, 065019.

Magin, R. L. (2006). Fractional Calculus in Bioengineering. Connecticut: Begell House.

Maurya, R. K., Devi, V., \& Singh, V. K. (2021). Stability and convergence of multistep schemes for 1D and 2D fractional model with nonlinear source term. Applied Mathematical Modelling, 89, 1721-1746.

https://doi.org/10.1016/j.apm.2020.08.038 
Moshrefi-Torbati, M., \& Hammond, J. K. (1998). Physical and geometrical interpretation of fractional operators. J. Franklin Inst., 335B, 1077-1086. https://doi.org/10.1016/S0016-0032(97)00048-3

Podlubny, I. (1999). Fractional Differential Equations. San Diego: Academic Press.

Qiu, W. L., Xu, D., Guo, J., \& Zhou, J. (2020). A time two-grid algorithm based on finite difference method for the two-dimensional nonlinear time-fractional mobile/immobile transport model. Numerical Algorithms, 85, 39-58. https://doi.org/10.1007/s11075-019-00801-y

Schumer, R., \& Benson, D. A. (2003). Fractal mobile/immobile solute transpport. Water Resources Research, 39, 12961308. https://doi.org/10.1029/2003WR002141

Singla, K., \& Gupta, R. K. (2016). On invariant analysis of some time fractional nonlinear systems of partial differential equations. I. Journal of Mathematical Physics, 57, 101504. https://doi.org/10.1063/1.4964937

Sun, Z. Z. (2018). The Difference Methods for Nonlinear Evolution Equations (in Chinese). Beijing: Science Press. https://doi.org/10.1515/9783110616064

Sun, Z. Z., \& Gao, G. H. (2020). Fractional Differential Equations-Finite Difference Methods. Beijing: Science Press and Berlin/Boston: De Gruyter.Xu, J. C. (1994). A novel two-grid method for nonlinear semilinear elliptic equations. SIAM J. Sci. Comput., 15, 231-237. https://doi.org/10.1137/0915016

Xu, J. C. (1996). Two-grid discretization techniques for linear and nonlinear PDEs. SIAM J. Numer. Anal., 33, 17591777. https://doi.org/10.1137/S0036142992232949

Wang, S. M., Zhang, M. J., \& Fan, X. M. (2018). Spatial dynamics of periodic reaction-difusion epidemic models with delay and logistic growth. Applied Mathematics and Mechanics, 39, 226-238.

Zhang, Y., Benson, D. A., \& Reeves, D. M. (2009). Time and space nonlocalities underlying fractional-derivative models: Distinction and literature review of field applications. Advances in Water Resources, 32, 561-581.

Zhao, X. Q. (2003). Dynamical Systems in Population Biology. New York: Springer-Verlag. https://doi.org/10.1007/9780-387-21761-1

Zhou, H. W., Yang, S., \& Zhang, S. Q. (2019). Modeling non-Darcian flow and solute transport in porous media with the Caputo-Fabrizio derivative. Applied Mathematical Modelling, 68, 603-615. https://doi.org/10.1016/j.apm.2018.09.042

\section{Copyrights}

Copyright for this article is retained by the author(s), with first publication rights granted to the journal.

This is an open-access article distributed under the terms and conditions of the Creative Commons Attribution license (http://creativecommons.org/licenses/by/4.0/). 